\title{
Review
}

Neuropsychobiology

Neuropsychobiology 2014;69:187-201

DOI: $\underline{10.1159 / 000360859}$
Received: July 23, 2013

Accepted after revision: February 19, 2014

Published online: May 23, 2014

\section{Advantages and Challenges of Small Animal Magnetic Resonance Imaging as a Translational Tool}

\author{
Carolin Hoyer $^{\mathrm{a}}$ Natalia Gass ${ }^{\mathrm{b}}$ Wolfgang Weber-Fahr ${ }^{\mathrm{b}} \quad$ Alexander Sartorius $^{\mathrm{a}, \mathrm{b}}$ \\ ${ }^{a}$ Department of Psychiatry and Psychotherapy, and ${ }^{b}$ Research Group Translational Imaging, Department of \\ Neuroimaging, Central Institute of Mental Health, Medical Faculty Mannheim, Heidelberg University, \\ Mannheim, Germany
}

\section{Key Words}

Animal models · Magnetic resonance imaging ·

Translational research

\section{Abstract}

The utilization of magnetic resonance imaging (MRI) methods in rodent models of psychiatric disorders provides considerable benefits for the identification of disease-associated brain circuits and metabolic changes. In this review, we discuss advantages and challenges of animal MRI and provide an overview of the major structural (voxel-based morphometry and diffusion tensor imaging) and functional approaches [resting-state functional MRI (rs-fMRI), MR spectroscopy (MRS), regional cerebral blood volume measurement and arterial spin labelling] that are applied in animal MRI research. The review mainly focuses on rs-fMRI and MRS. Finally, we take a look at some recent developments and refinements in the field.

\section{Animal Magnetic Resonance Imaging in Psychiatric Research}

Introduction

Preclinical animal models make valuable contributions to improving our understanding of human diseases, ranging from insights into the molecular and cellular underpinnings of a disorder to the development of novel pharmacotherapeutic treatment approaches. One of the main advantages of animal studies is group homogeneity, which cannot be easily achieved in clinical (human) studies. Yet, establishing valid animal models of psychiatric disorders has proven to be an undertaking of substantial proportions $[1,2]$. The pronounced heterogeneity of mental disorders and a strong reliance on phenomenology have impeded any precise evaluation of the validity of respective animal models [3]. One way to confront this problem has been to reverse-translationally adapt the concept of endophenotypes $[4,5]$ to preclinical research,

\section{KARGER}

E-Mail karger@karger.com www.karger.com/nps
C 2014 S. Karger AG, Basel

0302-282X/14/0694-0187\$39.50/0
Alexander Sartorius

Central Institute of Mental Health

Medical Faculty Mannheim, Heidelberg University, J5

DE-68159 Mannheim (Germany)

E-Mail alexander.sartorius@zi-mannheim.de 


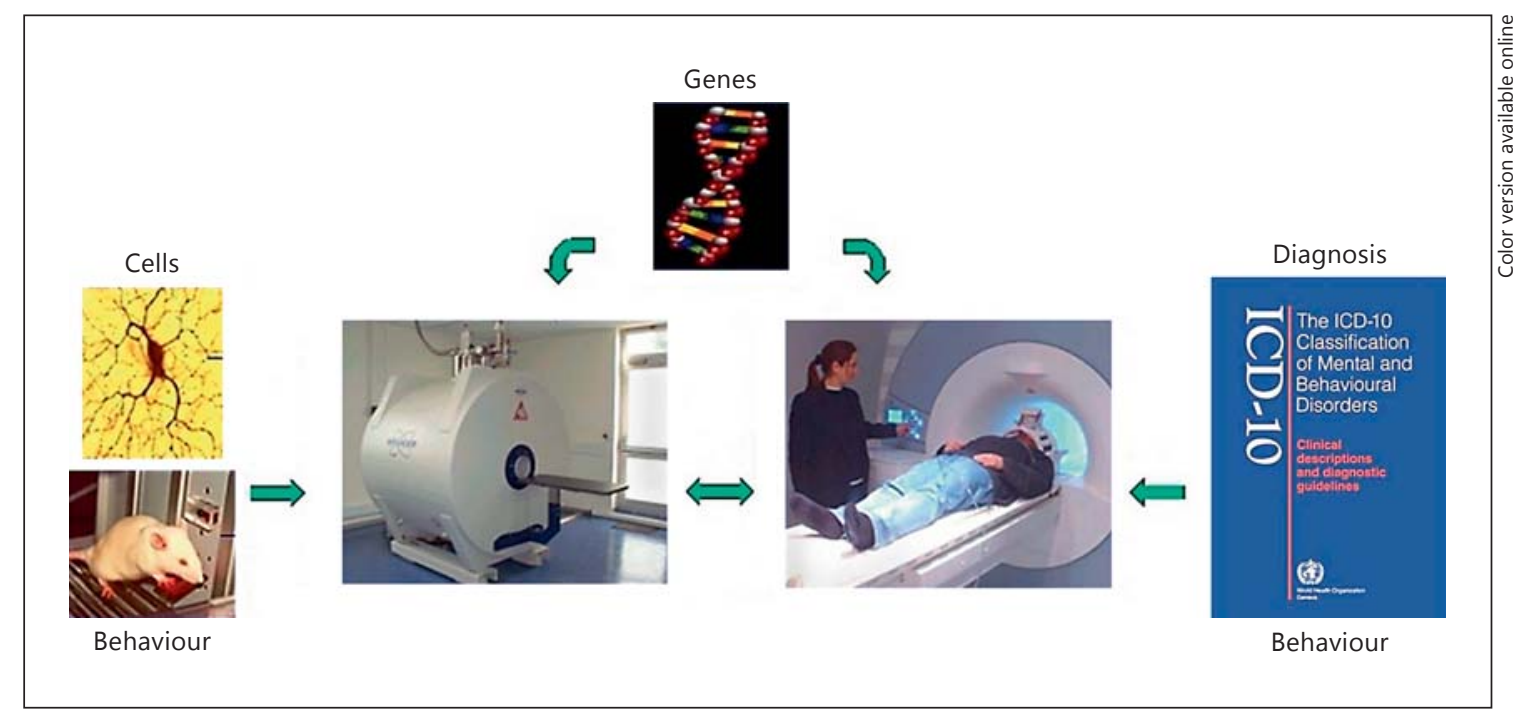

Fig. 1. Translational MRI-based research covers not only preclinical-to-clinical but also genotype-to-endophenotype mapping.

thereby emphasizing the dissection of functional circuits related to animal behaviour [2]. In this regard, neuroimaging is a highly valuable method in animal research; conceptually, it is informative with respect to the investigation of experimental manipulations shaping functional circuits and behaviour. Methodologically, the application of analogous imaging technology to animals and humans positively affects the translational potential of animal research (fig. 1).

Moreover, the last decade has seen the critical appraisal of a combined impact of genetic and environmental factors upon disease susceptibility [6]. Due to its complexity, the investigation of gene-by-environment(-by-treatment) interactions calls for an intermediate level of analysis. Since risk genes as well as environmental and pharmacological manipulations specifically influence brain functioning, high-resolution animal imaging provides a highly suitable technique to investigate these aspects in isolation or in their interaction. Finally, functional magnetic resonance imaging (fMRI) drives the progress of in silico technologies as are used in the Blue Brain Project (http:// www.humanbrainproject.eu/), the Human Brain Project (http://www.humanbrainproject.eu/) or the Bernstein Centres for Computational Neuroscience.

\section{Advantages of Non-Invasive Imaging Research:}

Repeated Measurements and Multimodality

Neuroimaging provides an excellent way to investigate structural and functional aspects of disease-associated pathology in animal models and clinical populations alike. Apart from this methodological advantage, non-invasive imaging as a research tool implements Russell and Burch's [7] classic '3R' principles of 'reduction', 'refinement' and 'replacement' for the humane treatment of research animals. Reduction can be achieved through smaller group sizes (reduced variance due to intra- and interindividual measurements). In addition, due to the possibility of performing repeated intraindividual measurements, the number of experimental groups can be reduced. High-resolution morphological and functional analyses as well as the use of methods causing as little distress as possible contribute to refinement.

An important feature of non-invasive procedures concerns the possibility of performing multimodal investigations on one and the same animal, allowing for a simultaneous or step-wise analysis of structure and function. In theory, a specific brain region may be investigated by voxel-based morphometry (VBM), resting-state fMRI (rs-fMRI), arterial spin labelling (ASL), diffusion tensor imaging (DTI) and MR spectroscopy (MRS). Impressive studies combining spectroscopy and fMRI have recently shown the great potential of a multimodal investigational approach to imaging research [8].

\section{Challenges and Limitations}

One of the biggest challenges of animal MRI concerns its resolution: while the human brain has an average volume of about $1,450 \mathrm{ml}$, that of a mouse is only $0.5-0.6 \mathrm{ml}$. 
Accordingly, while resolutions of about $1 \mathrm{~mm}$ suffice for most human brain imaging needs, the investigation of a rodent brain requires voxel sizes to be $0.1 \%$ of those required for human imaging. For MRS, in humans, the typical hippocampus voxel would contain approximately $3,000-4,000 \mu \mathrm{l}$, compared with $3-4 \mu \mathrm{l}$ in mice and 12 $15 \mu \mathrm{l}$ in rats. Since the signal-to-noise ratio (SNR) is directly proportional to the voxel size, this difference should be balanced in order to produce high-quality images.

\section{Limitations}

The SNR of a typical imaging application (e.g. MRS of the hippocampus) in a 3-Tesla human scanner exceeds that of a comparable animal measurement in a 9.4-Tesla scanner by a factor of 5 , which can only be balanced by repeated signal acquisition, thus necessitating longer durations of measurements; doubling the SNR, for instance, requires quadrupling the time of measurement.

Physiological noise is a much greater problem in animal imaging as well. Basically, the amount of movement a small animal induces through breathing (even under anaesthesia) is higher in relation to the voxel size in humans. Also, at a higher signal strength, physiological noise (breathing and cardiac pulse) fraction increases [9]. Due to the higher field power and resolution of animal scanners, physiological parameters may cause relevant disturbances. At best, they contribute noise to the acquired data; at worst, they cause strong artefacts, thereby seriously impairing image quality. Very sensitive DTI sequences in fact may be triggered by breathing in order to avoid or minimize artefacts.

For fMRI, a continuous recording of physiological activity is required at a sufficient temporal resolution of about $100 \mathrm{~Hz}$ sampling frequency. Since fMRI, especially in animals, always undersamples physiological noise, the frequencies of respiratory and cardiac noise may appear anywhere in the frequency band of interest due to aliasing effects. Using physiological curves recorded with high temporal resolution allows retrospective methods such as RETROICOR [10] to assess the influence of these distortions on the fMRI time series and to remove them by using regression methods.

For most applications of animal MRI, anaesthesia is a necessity. Even though it places restrictions on fMRI, experiments with awake animals also have their limitations [11]. For example, even after habituation to the apparatus, animals are still stressed by the fixation, which may subsequently have an impact on the investigated brain functions. So far, there has been a relative paucity of studies

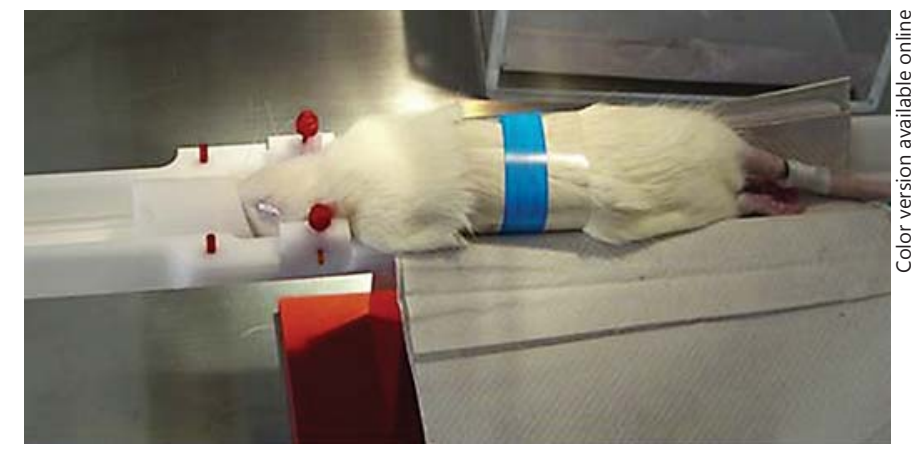

Fig. 2. A Sprague-Dawley rat on a small animal sleigh. The anaesthetic agent is supplied through the white tube. The eyes are protected by an ointment. The head is fixated with plastic pins positioned in the ear canal. The blue band (colours online only) indicates the breathing sensor. Body temperature is monitored through a rectally fixated probe.

looking at the effects of different types and depths of anaesthesia on cortical and subcortical functioning. Commonly, anaesthesia is achieved through the inhalative application of isoflurane, which is advantageous in different respects: it is usually well tolerated, and animals breathe spontaneously and do not require endotracheal intubation (fig. 2). Moreover, anaesthesia can be easily controlled by adjusting the isoflurane concentration. Rats usually receive $1.5-2 \%$ isoflurane, $30-50 \%$ oxygen and $50-70 \%$ air or $\mathrm{N}_{2} \mathrm{O}$; mice require slightly lower doses of isoflurane. The concentration may initially be higher and drop during the course of a longer experiment. However, isoflurane has major disadvantages for rs-fMRI studies, which are discussed in detail in the section titled 'Resting-State fMRI'.

\section{Field Strength}

Animal imaging systems often possess higher field powers and stronger gradient systems than human scanners. Higher-intensity fields are more favourable regarding the SNR (roughly linear) and allow for a higher spectral resolution in MRS. A 9.4-Tesla system increases the SNR by about a factor of 3 , which is actually countered in part by a disadvantageous change in relaxation parameters for imaging. The advantages of the higher field strength become really important for MRS, where not only the gain in SNR but also the higher spectral resolution improve the data. This means that overlapping resonance lines move further apart on the frequency axis and are easier to quantify. For imaging applications, this higher chemical shift is rather a disadvantage, since it could produce artefacts in the data (e.g. lipid artefacts in fast imaging sequences). 
Fig. 3. Schematic representation of VBM procedure. Representative, high-resolution 3D image data are segmented into different tissue components by a 2-step segmentation process. Using the DARTEL algorithm [61], they are registered to a group template, which is then used for the segmentation of the experimental data. GM = Grey matter.

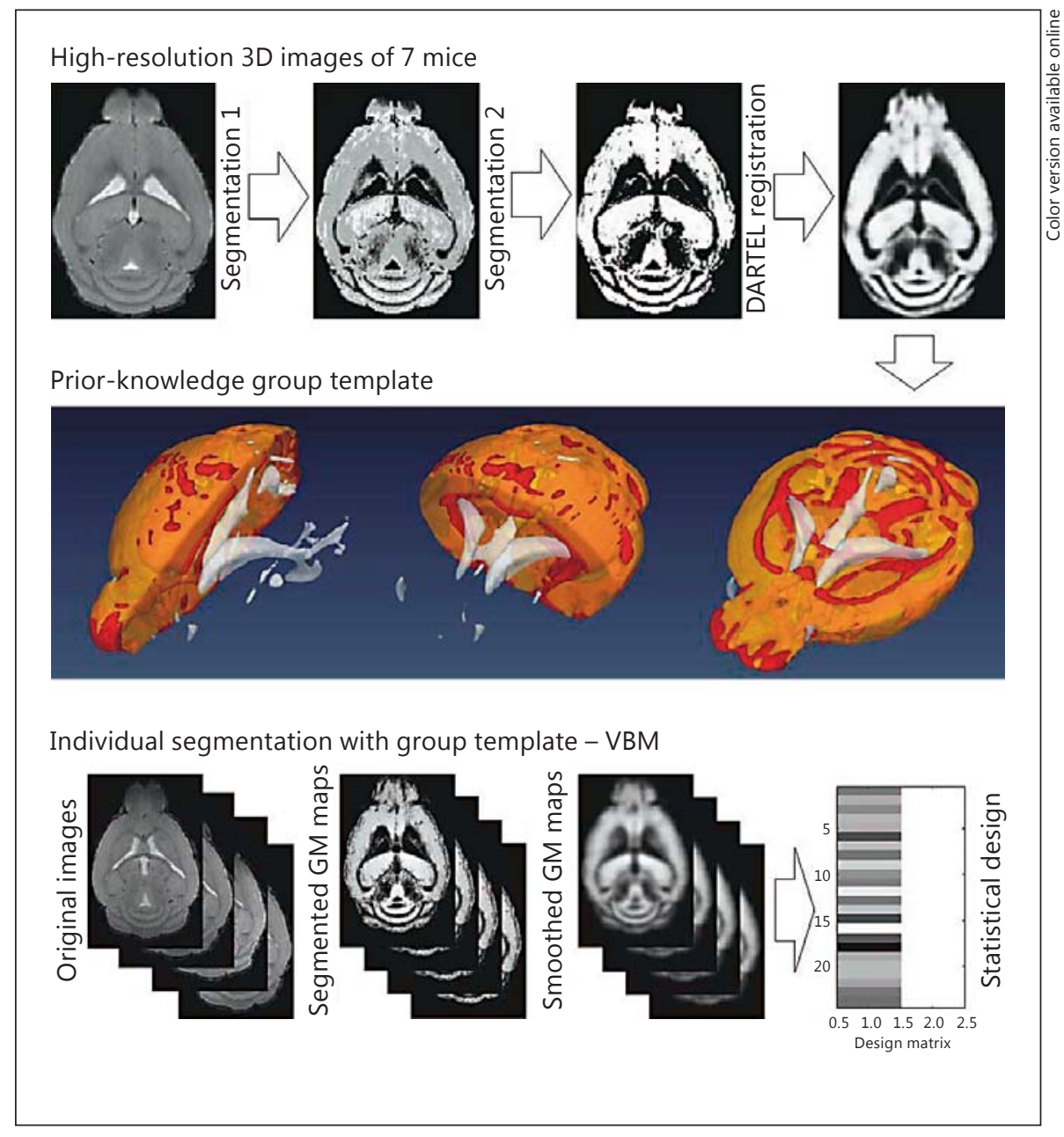

Apart from the changes in relaxation parameters, the most important disadvantages of a higher field strength are a higher magnetic field inhomogeneity and an increased specific energy absorption rate. High-field-strength scanners are more prone to the influence of disruptive artefactgenerating factors. Due to physical limitations, homogeneous static fields (B0) or radio frequency fields of the high-frequency coils (B1) are increasingly difficult to provide with field intensities of $7 \mathrm{~T}$ and above. However, a homogeneous B0 field is required for high-quality spectra and artefact-free images, and B1 inhomogeneities impede the quantification of MRS and imaging data. In order to balance B0 inhomogeneity, shim coils are applied. Field distortions depend on the measured object and require repeated field adjustments, which often do not yield the desired effect. MRS and fast imaging sequences such as echo planar imaging (EPI) used in fMRI and DTI are particularly sensitive in this regard. The increased specific energy absorption rate means that for the same application more energy will be absorbed by the tissue, which is a problem in human rather than in animal imaging.

\section{MR Coils}

The maximum increase in SNR in animal imaging is achieved by dedicated transmit-and-receive coils. Small radio frequency coils placed in close proximity to the animal can highly improve the quality of the acquired data. Recently, manufacturers introduced cryogenic coils cooled to low temperatures around $20-30 \mathrm{~K}$. This greatly reduces the noise generated by the coil itself and the preamplifier electronics and can thus improve the SNR by another factor of about 3 for protons and by much higher factors for other nuclei. In the case of larger animals and humans, the benefit from a cooled coil would be much smaller, since most of the noise would come from the much larger object of interest. 
Fig. 4. Areas with significant differences in grey matter volume induced by wheel-running in a murine hippocampus [62]. The colour scale shows the $T$ value of the statistical test.
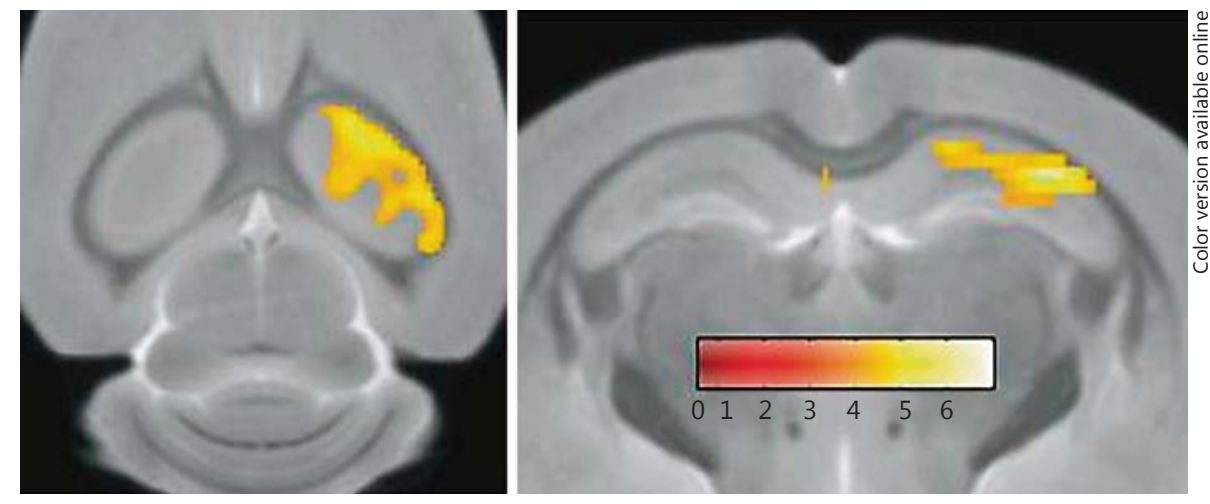

\section{Translational Structural Methods}

The emergence of high-resolution scanning technology and sophisticated image processing has significantly advanced structural analysis of the rodent brain. Endophenotype mapping as well as the assessment of the structural effects of ageing and environmental or pharmacological manipulations would considerably benefit from improved structural data.

Morphometric analysis can generally be carried out in two different ways. Regions of interest (ROIs) can be selected, which requires an a priori hypothesis or knowledge about structures potentially affected by an experimental factor. Methods like VBM or DTI, on the other hand, are based on semi-automatic procedures of tissue separation.

\section{Voxel-Based Morphometry}

In VBM [10], different anatomical attributes can be analysed and compared based on tissue contrast mapping due to MRI intensity differences. VBM is an unbiased method allowing a fine-grained structural analysis which, however, requires extensive processing of data. High-resolution $\mathrm{T}_{1^{-}}$or $\mathrm{T}_{2}$-weighted $3 \mathrm{D}$ datasets are partitioned into tissue classes (grey matter, white matter and cerebrospinal fluid) by segmentation algorithms. In this process, images are matched onto a common template with non-linear spatial normalization procedures, and the tissue probability maps are modulated with the local non-linear volume change [12]. The segmented and normalized images containing a semi-quantitative measurement of the local tissue concentrations are then smoothed so that each voxel has the average of grey matter from itself and its surroundings. Smoothing is done to reduce the impact of residual misregistration between subjects/ images and to lower the possibility of non-gaussian data.
Voxel-wise statistical testing is performed using generalized linear models, whereby local structural differences (in volume or concentration) can be identified. Figure 3 depicts the steps required for the creation of a group template for segmentation and subsequent individual segmentation and statistics.

The output of VBM is statistical map displaying regions with significant differences in volume or concentration of the investigated tissue (fig. 4). VBM is mainly a qualitative assessment of local differences in volume between groups. A semi-quantitative absolute volume measurement of a tissue class in a region can be conducted by summation of the signal intensities in the normalized segmented images. Each voxel has a probability of containing one of the three tissue classes multiplied by the Jacobian determinant of the vector field used to non-linearly normalize each individual dataset to the group template. This is a factor greater or smaller than 1 if the local volume is compressed or inflated during the normalization, respectively. The sum of all voxels over a region of the anatomical atlas in a normalized grey matter image thus approximates the total amount of grey matter in this region. However, depending on the normalization parameters, there could still be residual misregistrations in the final images; hence it has to be taken into account that there is a possible inaccuracy of quantitative volume assessment by VBM.

\section{Diffusion Tensor Imaging}

DTI is a technique for visualizing the (micro-)structural brain organization. Movement of water molecules is more rapid in the direction of fibre tracts and slower in the perpendicular plane; the measurement of this difference (diffusion anisotropy) can be used to make inferences about the presence, position and integrity of white matter tracts. 


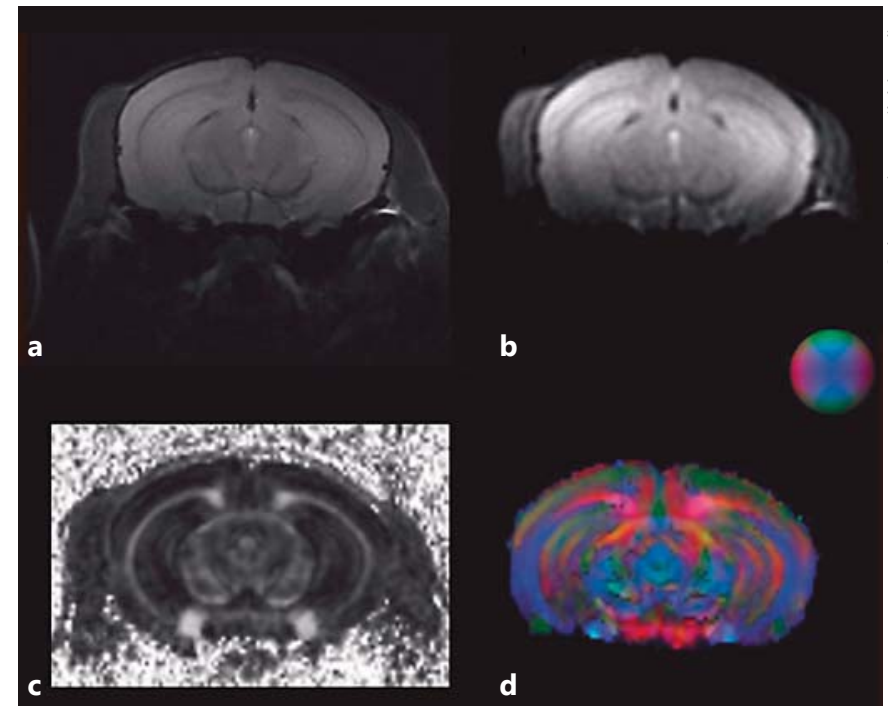

Fig. 5. DTI measurement of a murine brain. a Structural image. b Diffusion-weighted image at minimal diffusion time $(b=0)$. c Map of the fractional anisotropy calculated from the measured diffusion tensor. $\mathbf{d}$ Colour-coded map of the primary diffusion direction in the tensor. Blue: head-feet direction; red: left-right direction; green: anterior-posterior direction.

The diffusion tensor provides information about restricted and directed movements of water molecules with high spatial resolution. It is calculated from a comparison of MR signals acquired with diffusion gradients along at least 6 non-collinear, non-coplanar directions with MR signals without diffusion gradients. In practice, not only 6 but 32 or more diffusion measurements are taken in order to calculate a tensor of sufficient quality. This makes the technique time-consuming and prone to movement artefacts.

Essentially, two different processing methods are used for DTI. The simpler one is a calculation of the fractional anisotropy (FA) coefficient in every voxel, which states how directed the movement of water is in the current position compared with freely moving water. Since movement is restricted to one direction along white matter fibres, the resulting FA maps are particularly useful to measure changes in white matter. Therefore, DTI is often referred to as a white matter imaging method.

In the second method, the information from the calculated diffusion tensor is used to reconstruct white matter pathways in vivo. Tractography algorithms use this information to track the neural pathways based on the assumption that the dominant direction of water motion aligns with the fibre orientation in an imaged voxel. While this method produces striking visual presentations on fibre tracts, the results are not necessarily always accurate, since most algorithms are based on probability functions and are easily thrown off course by the presence of noise or artefacts in a single voxel along the tract. Also in voxels with fibre crossing the calculation of the diffusion tensor would represent a mean of the movement along all fibres present in the given voxel, thus impeding an estimation of the dominant direction in the given voxel by tractography algorithm.

Since the measurement of 32 or more diffusion directions is time-consuming, fast imaging sequences such as EPI are used for DTI. However, they are prone to geometrical artefacts and are highly sensitive to movement. Hence, most mouse brain DTI reports use data from ex vivo analysis of the brain, with only few attempts to perform in vivo DTI [13]. The movement problem can often be successfully solved by triggering the sequence to the animal's breathing cycle, which prolongs the time of measurement of one DTI dataset to about $1.5 \mathrm{~h}$. Figure 5 displays an example of data from a murine DTI experiment.

\section{Translational Functional Methods}

\section{Arterial Spin Labelling}

The translational application of functional imaging methods requires acknowledgement of the fact that most classic sensory stimulation paradigms cannot be used because of the need for animals to be anaesthetized. The blood oxygen level-dependent (BOLD) technique has long been the closest approximation to a functional analysis. Though well established and possessing a high degree of sensitivity, the BOLD effect is complex, and despite its dependence on cerebral blood flow, it does not give a direct measure of it. The direct and absolute quantification of cerebral blood flow requires a diffusible tracer. The most effective approach, called ASL, uses arterial blood water that is magnetically (spin) labelled by radio frequency irradiation. The change in tissue magnetization due to the labelled arterial blood is proportional to cerebral blood flow. In general, ASL has a rather poor SNR but does not require the application of contrast agents and is directly translational. It allows the assessment of task-independent measures of brain activation via the measurement of resting-state cerebral blood flow. Several translational ASL techniques exist which rapidly measure blood flow with improved SNR and high retest reliability [14], one of them being continuous ASL [15-17]. 


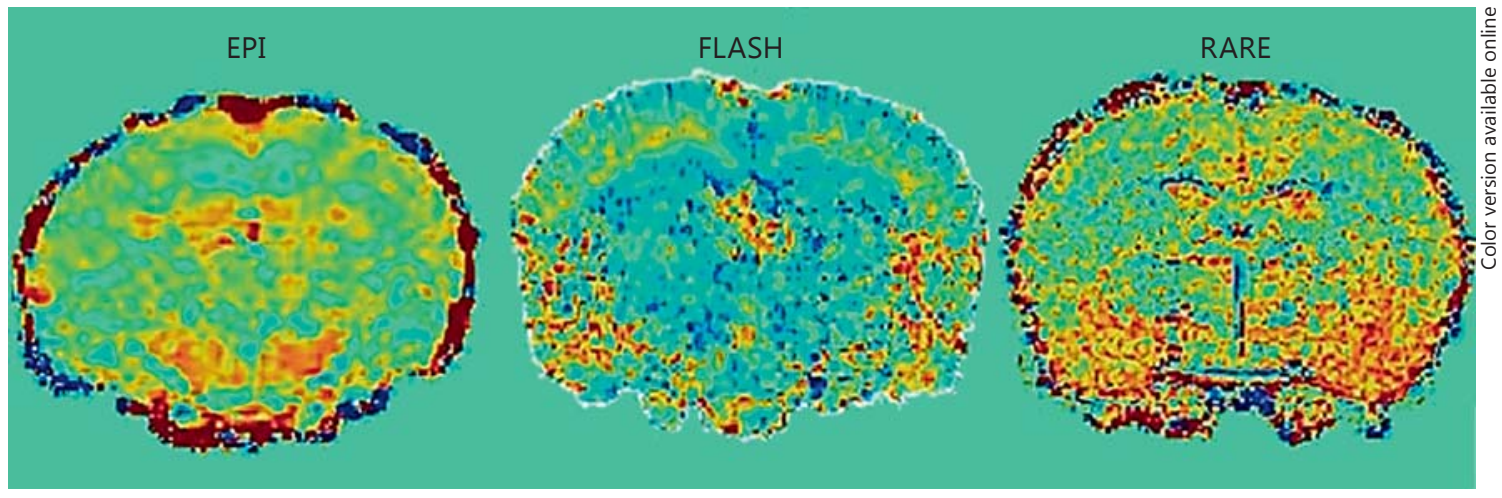

Fig. 6. $\mathrm{rCBV}$ images following the application of a gadolinium-based contrast agent (Omniscan; $10 \mathrm{mmol} / \mathrm{kg}$, i.p.) and a ketamine challenge $[12.5 \mathrm{mg} / \mathrm{kg}(\mathrm{S})$-ketamine]. Intraindividual reference measurements were taken after a 1-week washout phase. FLASH = Fast low-angle shot; RARE = rapid acquisition with relaxation enhancement.

\section{Regional Cerebral Blood Volume}

Regional cerebral blood volume (rCBV) measurements make use of the fact that the acute passage of a bolus of non-diffusible contrast agent through the brain causes a change in signal intensity that correlates with the regional blood volume. The mechanisms of contrast agents rely on their changes in local longitudinal relaxation time $\left(\mathrm{T}_{1}\right)$ and/or transverse relaxation time $\left(\mathrm{T}_{2}\right)$.

Several kinds of contrast agent are generally available for $\mathrm{rCBV}$ measurement in the rodent brain. On the one hand, there are gadolinium-based contrast agents with particle sizes smaller than $1 \mathrm{~nm}$, and on the other hand, several kinds of superparamagnetic iron oxide (SPIO) nanoparticles, which are based on magnetite $\left(\mathrm{Fe}_{3} \mathrm{O}_{4}\right)$ or maghemite $\left(\gamma-\mathrm{Fe}_{2} \mathrm{O}_{3}\right)$ and have particle sizes between 10 and $180 \mathrm{~nm}$. The large particle size originates from the coating of the paramagnetic molecules with polysaccharide or synthetic polymers or monomers. The smaller SPIO nanoparticles with a particle size below $50 \mathrm{~nm}$ - also called ultrasmall SPIO or USPIO particles - have a longer half-life in the blood after intravenous injection and are thus better suited for rCBV measurements than are larger particles. All contrast agents usually prolong the longitudinal $\mathrm{T}_{1}$ relaxation constant and shorten the transverse $\mathrm{T}_{2} / \mathrm{T}_{2}{ }^{*}$ relaxation times. USPIO particles have a stronger difference in these changes with an emphasis on shorter transverse relaxation times, and they have an excellent relaxivity as well as stronger effects than gadoliniumbased contrast agents on a per-atom basis. Quantification of the different effects produced by a contrast agent on the signal is complex and depends on vasculature size, field strength and pulse sequence; the $\mathrm{T}_{2}$ contrast from spin echo sequences, for instance, is sensitive to small vessels, while changes in $\mathrm{T}_{2}{ }^{*}$ which can be detected by gradient echo-based sequences are attributed more to larger vessels. When a contrast agent reaches a uniform distribution, relative CBV maps can be measured from steadystate $\mathrm{T}_{2}$-weighted images with the dependence $\mathrm{CBV} \propto$ $\Delta \mathrm{R}_{2}=\ln \left(\mathrm{S}_{\text {pre }} / \mathrm{S}_{\text {post }}\right) / \mathrm{TE}$, with the relaxivity $\mathrm{R}_{2}=1 / \mathrm{T}_{2}$, and $S_{\text {pre }}$ and $S_{\text {post }}$ as the signal intensities before and after contrast agent application.

While USPIO particles have a comparably strong effect on relaxation time and a long circulating half-life, they are more costly than gadolinium-based contrast agents and can lead to organ toxicity, which makes them not ideally suited for longitudinal studies. Gadoliniumbased agents, on the other hand, can repeatedly be applied intraperitoneally and have minimal toxicity [18]. They typically reach a steady state about 30-60 min after intraperitoneal injection, which normally lasts for approximately $1 \mathrm{~h}$. The kinetics depends on the nature of the contrast medium and its way of application. Regional blood volume measurements are well suited for pre-post comparisons in the context of pharmacological interventions [19]. Voxel-wise calculation of differences in rCBV intensity between the presence and absence of a pharmacological challenge allows a direct and non-invasive calculation of rCBV maps.

Different types of sequences can be used for quantifying changes in signal intensity after the application of a contrast agent. Figure 6 displays EPI, fast low-angle shot and rapid acquisition with relaxation enhancement sequences, where the EPI sequence captures agent accumulation at the highest temporal resolution. 

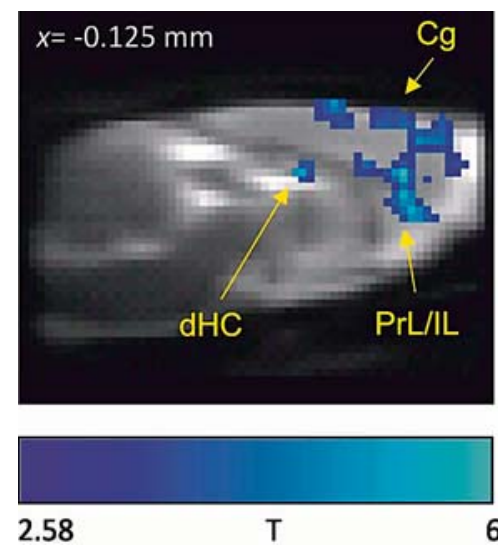
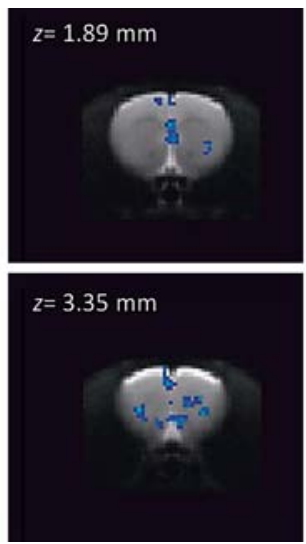
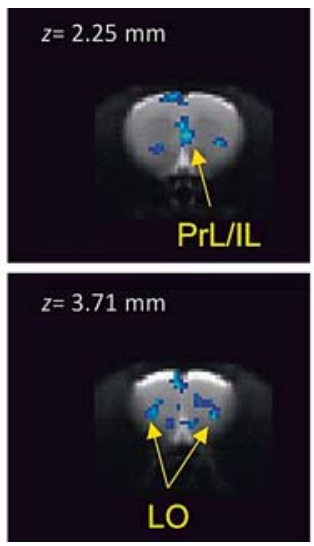
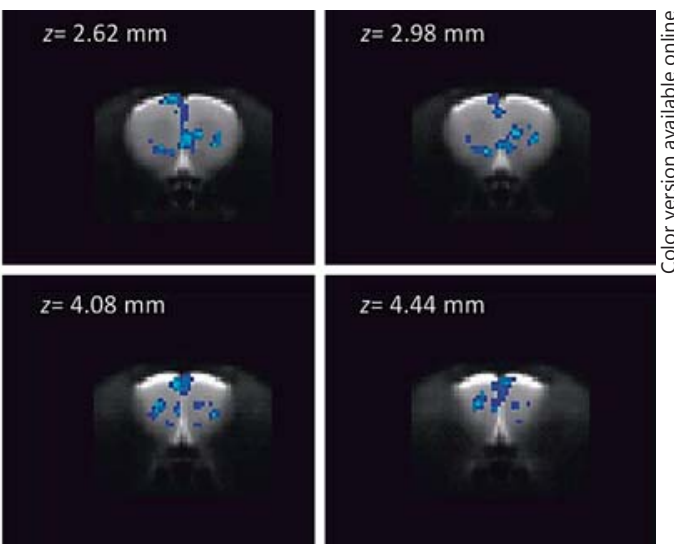

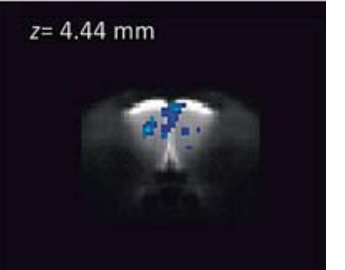

Fig. 7. Effect of haloperidol on voxel-wise functional connectivity with the substantia nigra. Blue: haloperidol < saline. The $\mathrm{z}$ coordinates are relative to the bregma; the $\mathrm{x}$ coordinate is relative to the midline. $\mathrm{Cg}=\mathrm{Cingulate}$ cortex; $\mathrm{dHC}=$ dorsal hippocampus; IL = infralimbic cortex; $\mathrm{LO}=$ lateral orbital cortex; PrL = prelimbic cortex [30].

Initial studies have been able to impressively demonstrate the potential of the rCBV method to boost highquality translational research: Small's group [20], for example, showed that baseline abnormalities in $\mathrm{rCBV}$ in the CA1 region of the hippocampus of prodromal patients were predictive of the development of psychosis. In the same study, rCBV mapping was performed on mice receiving antipsychotics, and the antipsychotic medication could be excluded as a potential confounder of the study results [20].

\section{Resting-State fMRI}

Although the first report on human brain functional connectivity in the absence of any stimulus or task was already published as far back as 1995 [21], only recently have resting states also been described for the rodent brain. One of the main reasons why it took longer to establish them in animals is the requirement for anaesthesia and physical restraint in the scanner. However, low-frequency $(<0.1 \mathrm{~Hz})$, temporally synchronized BOLD oscillations reflecting functionally connected brain networks analogous to humans were demonstrated in anaesthetized rodents [22-28]. The observed networks relate to sensorimotor, hippocampal, prefrontal, retrosplenial, visual, auditory, caudate-putamen and thalamic networks. It has been suggested that anaesthetized rat brains also possess a default mode network that is comparable to that of humans and includes hippocampal, prefrontal, cingulate, posterior parietal and retrosplenial cortices [29]. Therefore, these coherent BOLD fluctuations may represent a principal intrinsic property of a functioning brain and can be used as a valuable preclinical tool in animal models of disease to investigate diagnostic markers in the form of changes in functional connectivity. In addition, the effect of pharmacological manipulations of functional connectivity can be studied preclinically (fig. 7) [30].

Resting-state networks have also been reported in nonanaesthetized rats [31-35]. Before conducting experiments, the animals are habituated to the restrained position; typically, they are lightly anaesthetized and placed into a mock scanner with scanner noises simulating the future experimental set-up. The number of acclimation sessions has varied in different studies (from 2-3 days [31, $34]$ to 8 days $[32,35])$. However, these studies are still few in number due to their main limitation, i.e. the stress animals have to undergo in the restrained position in the scanner and its consequential effect on brain functional connectivity patterns, and also due to their need for several habituation sessions. Interestingly, a recent study has shown that although an anaesthetized rat brain (under isoflurane) displays a weaker connectivity, it still preserves global network properties of the awake brain (in terms of graph theory: global clustering coefficient, mean shortest path length, small-worldness and modularity), and longdistance connections are not preferentially reduced [33]. A recent study on anaesthetized human brains (under propofol) exploring functional connectivity in terms of graph theory has also shown that long-range and short connections are not differentially affected by anaesthesia, and the small-world architecture of the brain networks persists (is in fact increased); clustering and modularity are elevated, possibly indicating an increase in localized processing and lower integration [36]. 
The usual choice of anaesthetic has been isoflurane, because narcosis can be easily adjusted and kept stable for hours. However, the $\alpha_{2}$-adrenoreceptor agonist medetomidine was demonstrated to provide functional connectivity maps more comparable to what is seen in humans [37]: under medetomidine, more localized correlations were observed, in contrast to the widely spread connectivity across the cortex seen under isoflurane - which is possibly due to the latter's interference with neural activity, which medetomidine does not affect [38]. Medetomidine allows longitudinal experiments and does not require invasive procedures, in contrast to $a$-chloralose, which necessitates intubation and mechanical ventilation of animals. Functional connectivity maps from rats anaesthetized with $\alpha$-chloralose are more similar to the ones observed under medetomidine than those seen under isoflurane; however, they exhibit localization to a lesser extent [37]. Therefore, medetomidine is currently the preferred choice [23, 26, 28, 39-41].

Once an animal is anaesthetized and placed into the scanner, it undergoes a typical rs-fMRI experiment similar to the one on humans. Preferably, fast EPI sequences acquire data during a period of approximately $10 \mathrm{~min}$ with a repetition time of $1-3 \mathrm{~s}$. Since geometrical artefacts and the influence of physiological noise are bigger at a higher field strength, the $\mathrm{B} 0$ field maps and physiological signals (respiration and cardiac pulse) are sampled during the experiment. The cardiorespiratory cycle and variations in respiratory volume/rate may inflate ROI-ROI correlations and bury the real ones. Cardiac noise mostly affects brain regions bordering blood vessels, whereas respiratory motion can be detected in areas near the edges of the brain or near the ventricular system due to shifts in B0 from abdominal movement. Since both cardiac and respiratory motion usually occur at frequencies higher than the fMRI sampling frequency, their effects on the signal are aliased to lower frequencies possibly within the range of the resting-state fluctuations and cannot be simply filtered out by band-pass filtering. Thus, during data preprocessing, retrospective methods estimate the effect of the noise on the fMRI signal and regress it out of the data $[10,42]$. A resting-state network analysis usually concentrates on the spontaneous BOLD fluctuations in a frequency range between 0.01 and $0.1 \mathrm{~Hz}[21,43]$. However, recent studies have demonstrated that meaningful information is also contained at higher frequencies (at 0.17 and $0.25 \mathrm{~Hz}$ and higher) [44-46]. In the emerging field of rs-fMRI, numerous methods for detecting and representing these fluctuations have become available. One of the common methods is independent component

Advantages and Challenges of Small Animal MRI analysis, which looks for independent spatio-temporal modes to detect areas belonging to a synchronized network (fig. 8). In seed-based analysis, temporal correlations between distinct brain regions are calculated voxeland/or ROI-wise, offering a way of functional connectivity quantification. This analysis may be expanded by graph-theoretical methods to investigate network models over the selected brain areas, allowing a characterization of network-intrinsic properties. Further analytical approaches include clustering, pattern classification and several 'local' methods; their pros and cons are thoroughly reviewed e.g. by Margulies et al. [47].

\section{Translational MRS}

MRS is a unique in vivo method for investigating metabolic processes by detecting and quantifying in situ concentrations of certain chemical substances in the brain. MRS involves the detection of signals of compounds other than water by making use of the fact that certain atomic nuclei, if placed in a strong, static magnetic field, can interact with an externally applied, pulsed magnetic field, resulting in a frequency-specific resonance. Nuclei of different molecules possess distinct resonance frequencies and can be identified and distinguished on this basis.

\section{Single-Voxel MRS}

For single-voxel MRS, a cuboid-shaped target ROI is activated, and a single spectrum representing the biochemical composition of the ROI is measured. Spatial localization is achieved by sequential activation of three orthogonal layers, the intersection volume of which equals the ROI. An ideal ROI contains only tissue of interest and is of small size. The spatial resolution, however, is limited because MRS signals are generally quite weak and may easily disappear in the background noise if in vivo concentrations are very low. It is generally possible to compensate for signal loss by repeated signal acquisition; however, a $50 \%$ reduction in ROI size requires an extension of the duration of a measurement by a factor of 4 in order to achieve a similar SNR. Established sequences for single-voxel MRS analyses are the point-resolved spectroscopy sequence, consisting of one 90-degree and two 180-degree high-frequency pulses, and the stimulated echo acquisition mode sequence, consisting of three 90-degree high-frequency pulses.

Figure 9 displays an exemplary ${ }^{1} \mathrm{H}$ spectrum from a murine hippocampus indicating characteristic peaks of different metabolites. The area under a metabolite reso- 


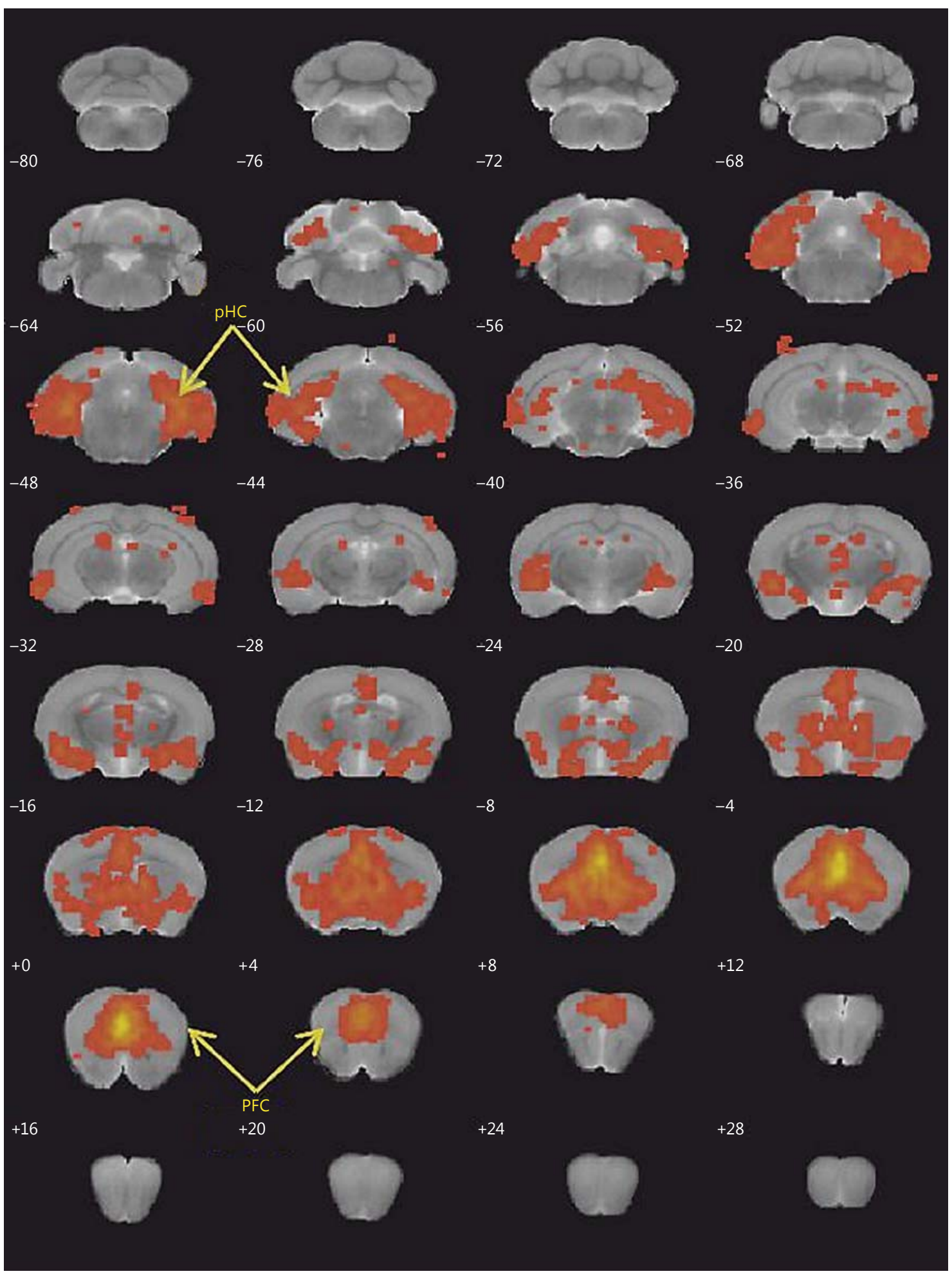

Fig. 8. Hippocampal-prefrontal resting-state functional connectivity network in mice. This network was identified by independent component analysis. $\mathrm{PFC}=$ Prefrontal cortex; $\mathrm{pHC}=$ posterior hippocampus. 
Fig. 9. ${ }^{1} \mathrm{H}$ spectrum of a murine hippocampus $(3.2 \mu \mathrm{l}$; point-resolved spectroscopy sequence; $\mathrm{TE}=10 \mathrm{~ms}$, $\mathrm{TR}=4 \mathrm{~s}$, average over 256 measurements). The red line indicates the result of the fitting function. The most prominent resonance peaks are $\mathrm{N}$-acetylaspartate (NAA), creatine and phosphocreatine $(\mathrm{Cr})$, choline-containing molecules (Cho), myo-inositol (mI), glutamate (Glu), glutamine (Gln) and their combination (Glx), taurine (Tau) and $\gamma$-aminobutyric acid (GABA). Asp = Aspartate.

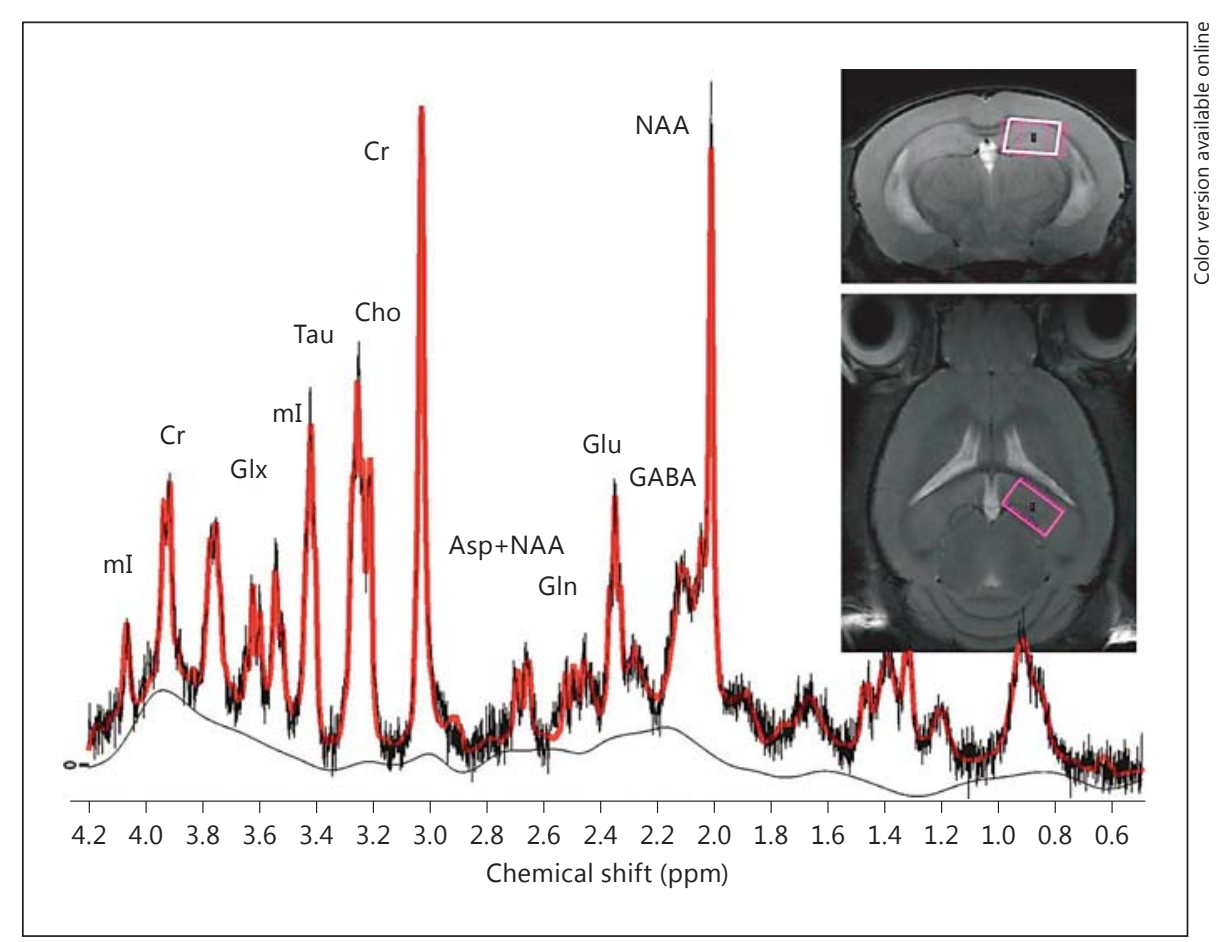

nance in the spectrum is proportional to the concentration of the metabolite within the measured volume. The integration of peak areas is usually sufficient in high-resolution MRS, since the peaks are clearly separated and the baseline does not fluctuate. In vivo MR spectra, however, often do not meet these prerequisites, and both the dominant water resonance in ${ }^{1} \mathrm{H}$-labelled spectroscopy and signals of slowly moving molecules may lead to an overestimation of the peak areas. In addition, the spectral resolution, i.e. the ability to distinguish adjacent peaks, is limited. Moreover, scalar coupling of different nuclei, indicative of molecular connectivity, frequently occurs and causes a splitting of the nuclear MR signal into multiplets with different intensity ratios.

As a consequence, there is a multitude of methods for in vivo MRS whereby the time or frequency signal is sought to be manipulated by mathematical operations $[48,49]$. For example, more recent procedures utilize a priori information about the composition of the spectrum to be measured which is derived from simulations or measurements of each substance in model solutions. The signal intensity of the resonance lines can then be calculated from parameters of the adapted model functions. The resulting intensity values, however, are not true representations of metabolite concentrations, due to the influence of hardware- and sequence-specific parameters

Advantages and Challenges of Small Animal MRI and the metabolites' relaxation times. Moreover, the volumes feeding into the spectra usually do not encompass just one tissue, eventually leading to the measurement of metabolite concentrations of different types of tissue. Segmenting the MRS volume elements into grey matter, white matter and cerebrospinal fluid and entering their values into the analysis as covariates can be helpful in this regard [50]. Most problems can be avoided by reporting intensity ratios rather than absolute values. For example, the MR signal of creatine is often used as a reference in ${ }^{1} \mathrm{H}$-labelled spectroscopy. However, this technique is only valid and useful if the concentration of one metabolite is assumed to be constant $[51,52]$.

Animal MRS benefits in two ways from the high field intensities used in modern animal scanners: the SNR and spectral resolution are higher, which means that overlapping resonance lines can be separated more easily. Particularly the distinction and identification of glutamate, glutamine and $\gamma$-aminobutyric acid (GABA) in animal scanners is easier and of superior quality compared with the performance of human scanners, which usually have lower field intensities of 1.5-3 Tesla

\section{In vivo ${ }^{1} \mathrm{H}-\mathrm{MRS}$}

Besides tritium, a hydrogen isotope only rarely found in nature, protons are the most sensitive nuclei in MRI. 

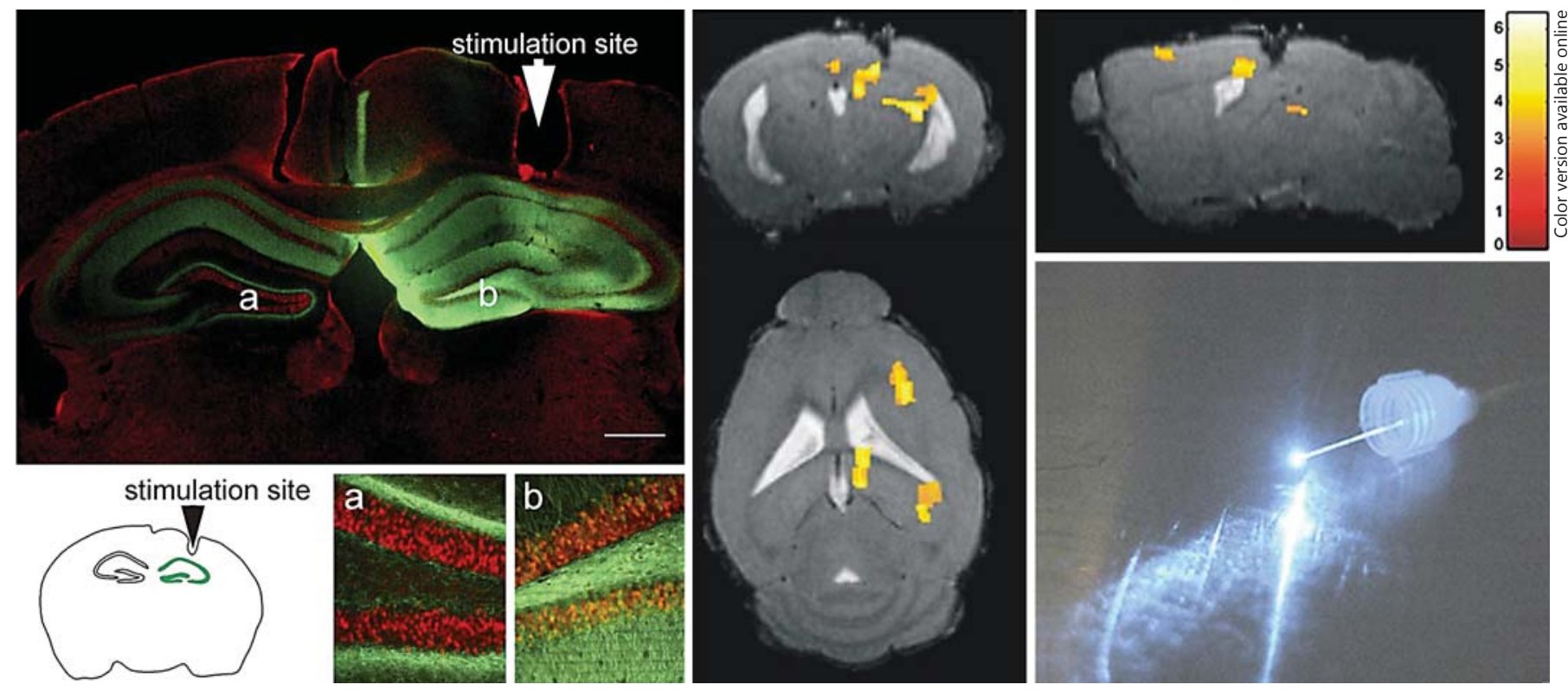

Fig. 10. Activation pattern in opto-fMRI after hippocampal channelrhodopsin transfection in CaMKIIa-Cre mice.

They are easy to measure due to their high prevalence and high gyromagnetic ratio. With a concentration of up to 40 $\mathrm{mol} / \mathrm{l}$, water is the most frequently occurring proton-containing molecule in live tissue. Even though its MR signal is the basis for MRI, it is a noise generator in MRS due to its much (up to 1,000-fold) higher intensity in comparison with other MR-detectable metabolites, requiring either suppression of the water signal during data acquisition or removal from the spectra in postprocessing [53].

Protons are part of most organic molecules, and the range of chemical shift is rather small. Thus, one would assume that in vivo ${ }^{1} \mathrm{H}$ spectra consist of a large number of overlapping lines. However, the resonances of only a few metabolites are directly detectable in ${ }^{1} \mathrm{H}$ spectra, for two reasons: (1) only small and mobile molecules generate a signal of sufficient intensity for detection and (2) a metabolite's concentration must be high enough (1-2 $\mathrm{mmol} / \mathrm{l}$ ) to be separable from the background noise.

Figure 9 displays the most important metabolites detectable by ${ }^{1} \mathrm{H}$-MRS. $\mathrm{N}$-acetylaspartate (NAA) is a neuronal marker, and the intensity of the NAA signal is a highly sensitive marker for morphologically intact and functioning neurons. The in vivo resonance of NAA consists of overlapping signals of NAA and, to a smaller extent, $\mathrm{N}$-acetylaspartylglutamate. Both signals can be separated only in a highly homogeneous field.

Even though occurring in smaller concentrations than NAA, choline-containing molecules have a high reso- nance at $3.22 \mathrm{ppm}$ due to their 3 methyl groups containing 9 magnetically equivalent protons. Choline emits further signals with a multiplet structure at 3.54 and 4.05 ppm - which, however, cannot be separated from the signals of other metabolites with lower intensity in vivo. The choline signal represents the choline pool. Acetylcholine, glycerophosphocholine and phosphatidylcholine - the latter two being integral cell membrane components [54] - are the functionally most important molecules of this pool. Increases in the choline signal indicate increases in membrane turnover, neuronal plasticity or reactive glia due to neuronal repair or degeneration.

Creatine and phosphocreatine form another group of important resonance signals. Creatine is partly ingested through food and partly synthetized in the liver, kidneys and pancreas from arginine, glycine and 5-adenosylmethionine. Phosphocreatine is the storage form of chemical energy in muscle, brain and nerve tissue [52]. Resonances of creatine and phosphocreatine are indistinguishable in in vivo ${ }^{1} \mathrm{H}$ spectra. Protons of the $\mathrm{CH}_{3}$ group are represented by a resonance signal at $3.02 \mathrm{ppm}$.

Myo-inositol (mI) is most easily detectable through its resonance at $3.54 \mathrm{ppm}$. The substance participates in intracellular signalling via inositol phosphate; however, the pathophysiological relevance of increased cerebral $\mathrm{mI}$ is largely unknown. In newborns, $\mathrm{mI}$ concentrations are significantly higher and decrease over several weeks. Further, $\mathrm{mI}$ is a sensitive marker for Alzheimer's disease [55]. 

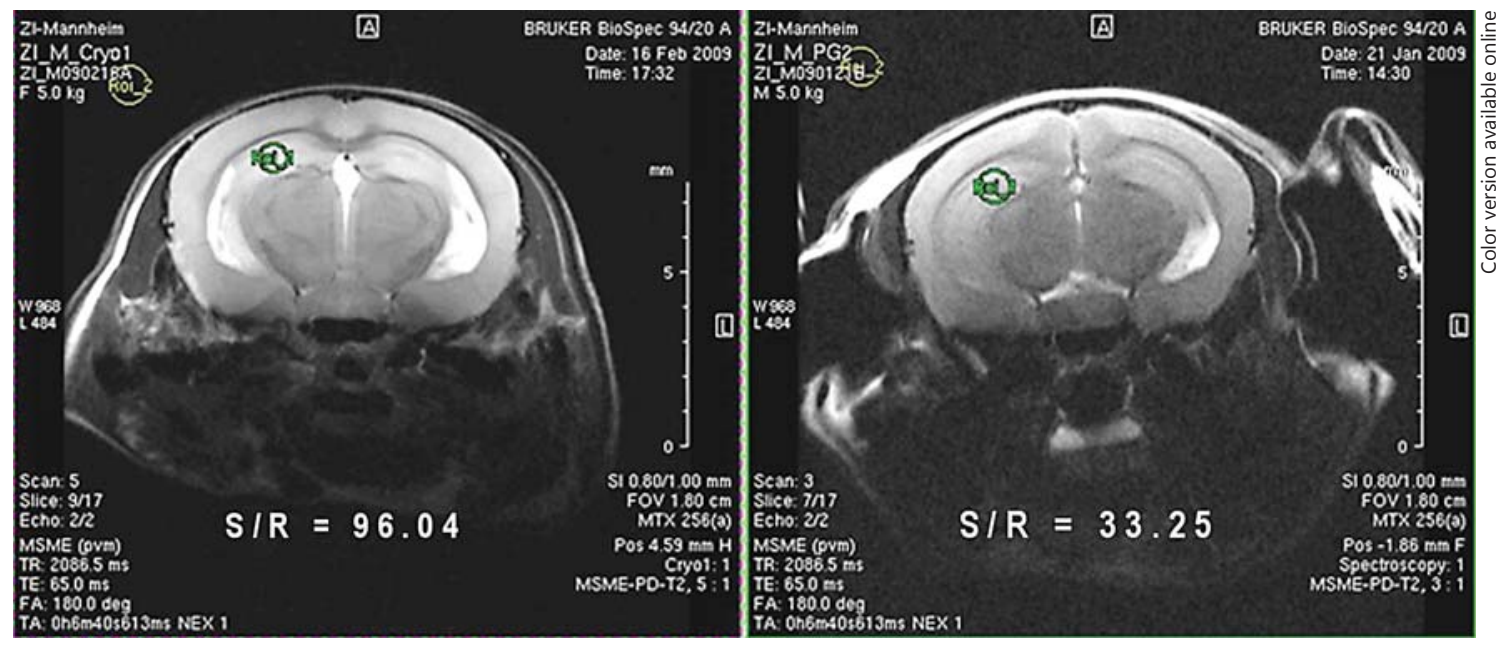

Fig. 11. Signal enhancement in an image of the murine brain by use of a cryogenic coil (left) compared with a standard 4-channel coil (right).

Glutamate is the most important excitatory amino acid. After synaptic release, it is metabolized to glutamine in the glia. Due to their complex multiplet structure, the resonances of glutamate and glutamine can only be separated in high field intensities (from 7 Tesla onwards). GABA can also be quantified at 7 Tesla. A change in the glutamate/GABA ratio may indicate a change from neurotoxicity to neuroprotection [54].

\section{Non-Translational Methods}

\section{Optogenetic fMRI}

An essential focus of psychiatric research lies on the investigation of functional networks within the brain. For example, prefrontal/hippocampal network connections are relevant in a variety of disorders such as depression or schizophrenia [56-58]. It is hitherto unknown how interregional interaction or functional connectivity alters under conditions of activation of specific groups of neurons. The combination of high-field MRI with optogenetic methods makes a pioneering move into investigating this question.

Optogenetics is a type of neuromodulation technology based on the specific expression of light-sensitive transmembrane conductance regulators such as channelrhodopsin-2, a monovalent $\mathrm{Na}$ conduction ion channel [59]. The activity of neurons expressing channelrhodopsin can be regulated directly in the range of milliseconds by application of blue light of $472 \mathrm{~nm}$ wavelength, which can be directed to a specific region in vivo by laser beam- transducing glass fibres. Both interneurons and excitatory neurons can be activated in a highly selective manner. Lee et al. [60] were the first to successfully describe the combination of optogenetics and functional imaging (opto-fMRI): rhodopsin was specifically activated in CaMKIIa-expressing excitatory neocortical and thalamic neurons and elicited BOLD signals not only locally but also in downstream targets of the stimulation sites. An example from our own group demonstrating the effect of CaMKIIa-expressing excitatory hippocampal neurons is displayed in figure 10 .

Opto-fMRI offers a huge potential regarding the causal identification and analysis of functional networks and endophenotyping of dysfunctional disease-associated circuits. It could further facilitate understanding of the therapeutic effects of non-specific deep brain stimulation.

\section{Further Refinements: Cryogenic Coils, X Nuclei, Manganese-Enhanced MRI}

A promising avenue regarding the improvement of SNR and thus image quality is the use of cryogenic coils in animal scanners. Both the coil and the preamplifier of the signal receiver are helium cooled at approximately $20 \mathrm{~K}$, which eliminates a large part of the thermal noise of the coil and electronic equipment. Cryogenic coils are mainly used in mouse scanners as bigger animals are a significant source of noise themselves. In mice, with ${ }^{1} \mathrm{H}$ labelled imaging techniques, an improvement in SNR by a factor 2.5 or higher is possible (fig. 11). In nuclei with lower frequencies (e.g. ${ }^{13} \mathrm{C}$ ), the improvement can be even more substantial. 
MRS allows the measurement of a growing number of different isotopes (X nuclei) besides protons, such as phosphor $\left({ }^{31} \mathrm{P}\right)$, carbon $\left({ }^{13} \mathrm{C}\right)$, lithium $\left({ }^{7} \mathrm{Li}\right)$, fluoride $\left({ }^{19} \mathrm{~F}\right)$ or sodium $\left({ }^{23} \mathrm{Na}\right)$, each possessing specific technological requirements (coil, high-frequency generator and sequences) and preferred areas of use. For example, lithium, with its scarce natural occurrence, is particularly suitable for the analysis of brain metabolic or pharmacokinetic features. Through quantifying ADP and ATP, phosphorus spectroscopy can be employed in the investigation of energy metabolism. Carbon spectroscopy could play a relevant role in the future of tracer technology as ${ }^{13} \mathrm{C}$ is a stable, non-radioactive isotope occurring in nature in minimal quantities (1.1\%). Compared with micro-PET, theoretically there is a much larger repertoire of tracers, since no radiochemical synthesis is needed.

Manganese-enhanced MRI (MEMRI) has been used for nearly two decades in the investigation of biological systems with the aims of contrast enhancement, assessment of neuronal activity and tract tracing. Manganese is a paramagnetic calcium analogue; depending on the place of injection (intracerebral, intraventricular or intraperitoneal), the anatomical disposition and excitability as well as the neural networks and their functional connectivity can be displayed in a unique and distinct manner. MEMRI is advantageous for animals as they can freely move 24-48 h after injection; neuronal activity recorded by MEMRI, provided by accumulation of manganese, is anaesthesia free. On the other hand, manganese is relatively neurotoxic, which is why often only individual injection attempts are made, making the use of automated analysis difficult.

\section{Conclusion}

To conclude, MRI methods have proven their high reliability and sensitivity in translational research and build an important link between basic and clinical research. A number of discussed issues and pitfalls are to be considered and improved, which can then further amplify the potential of rodent translational imaging studies.

\section{References}

1 Belzung C, Lemoine M: Criteria of validity for animal models of psychiatric disorders: focus on anxiety disorders and depression. Biol Mood Anxiety Disord 2011;1:9.

$\checkmark 2$ Berton O, Hahn CG, Thase ME: Are we getting closer to valid translational models for major depression? Science 2012;338:75-79.

3 Nestler EJ, Hyman SE: Animal models of neuropsychiatric disorders. Nat Neurosci 2010;13: 1161-1169.

4 Gottesman II, Gould TD: The endophenotype concept in psychiatry: etymology and strategic intentions. Am J Psychiatry 2003;160:636-645.

$\checkmark 5$ Meyer-Lindenberg A, Weinberger DR: Intermediate phenotypes and genetic mechanisms of psychiatric disorders. Nat Rev Neurosci 2006;7:818-827.

6 Caspi A, Moffitt TE: Gene-environment interactions in psychiatry: joining forces with neuroscience. Nat Rev Neurosci 2006;7:583-590.

7 Russell WMS, Burch RL: The Principles of Humane Experimental Technique. London, Methuen, 1959.

8 Walter M, Henning A, Grimm S, Schulte RF, Beck J, Dydak U, Schnepf B, Boeker H, Boesiger P, Northoff G: The relationship between aberrant neuronal activation in the pregenual anterior cingulate, altered glutamatergic metabolism, and anhedonia in major depression. Arch Gen Psychiatry 2009;66:478-486.

-9 Kruger G, Kastrup A, Glover GH: Neuroimaging at $1.5 \mathrm{~T}$ and $3.0 \mathrm{~T}$ : comparison of oxygenation-sensitive magnetic resonance imaging. Magn Reson Med 2001;45:595-604.
10 Glover GH, Li TQ, Ress D: Image-based method for retrospective correction of physiological motion effects in fMRI: RETROICOR. Magn Reson Med 2000;44:162-167.

11 Berwick J, Martin C, Martindale J, Jones M, Johnston D, Zheng Y, Redgrave P, Mayhew J: Hemodynamic response in the unanesthetized rat: intrinsic optical imaging and spectroscopy of the barrel cortex. J Cereb Blood Flow Metab 2002;22:670-679.

12 Ashburner J, Friston KJ: Voxel-based morphometry: the methods. Neuroimage 2000;11: 805-821.

13 Harsan LA, Paul D, Schnell S, Kreher BW, Hennig J, Staiger JF, von Elverfeldt D: In vivo diffusion tensor magnetic resonance imaging and fiber tracking of the mouse brain. NMR Biomed 2010;23:884-896.

14 Chen Y, Wang DJ, Detre JA: Test-retest reliability of arterial spin labeling with common labeling strategies. J Magn Reson Imaging 2011; 33:940-949.

15 Bruns A, Kunnecke B, Risterucci C, Moreau JL, von Kienlin M: Validation of cerebral blood perfusion imaging as a modality for quantitative pharmacological MRI in rats. Magn Reson Med 2009;61:1451-1458.

$>16$ Roiser JP, Levy J, Fromm SJ, Nugent AC, Talagala SL, Hasler G, Henn FA, Sahakian BJ, Drevets WC: The effects of tryptophan depletion on neural responses to emotional words in remitted depression. Biol Psychiatry 2009;66: 441-450.
17 Viviani R, Sim EJ, Lo H, Beschoner P, Osterfeld N, Maier C, Seeringer A, Godoy AL, Rosa A, Comas D, Kirchheiner J: Baseline brain perfusion and the serotonin transporter promoter polymorphism. Biol Psychiatry 2010;67:317322 .

18 Moreno H, Hua F, Brown T, Small S: Longitudinal mapping of mouse cerebral blood volume with MRI. NMR Biomed 2006;19:535-543.

19 Gozzi A, Large CH, Schwarz A, Bertani S, Cres$\tan \mathrm{V}$, Bifone A: Differential effects of antipsychotic and glutamatergic agents on the phMRI response to phencyclidine. Neuropsychopharmacology 2008;33:1690-1703.

20 Schobel SA, Lewandowski NM, Corcoran CM, Moore H, Brown T, Malaspina D, Small SA: Differential targeting of the CA1 subfield of the hippocampal formation by schizophrenia and related psychotic disorders. Arch Gen Psychiatry 2009;66:938-946.

21 Biswal B, Yetkin FZ, Haughton VM, Hyde JS: Functional connectivity in the motor cortex of resting human brain using echo-planar MRI. Magn Reson Med 1995;34:537-541.

22 Lu H, Zuo Y, Gu H, Waltz JA, Zhan W, Scholl CA, Rea W, Yang Y, Stein EA: Synchronized delta oscillations correlate with the restingstate functional MRI signal. Proc Natl Acad Sci USA 2007;104:18265-18269.

23 Pawela CP, Biswal BB, Cho YR, Kao DS, Li R, Jones SR, Schulte ML, Matloub HS, Hudetz AG, Hyde JS: Resting-state functional connectivity of the rat brain. Magn Reson Med 2008; 59:1021-1029. 
-24 Kannurpatti SS, Biswal BB, Kim YR, Rosen BR: Spatio-temporal characteristics of low-frequency BOLD signal fluctuations in isofluraneanesthetized rat brain. Neuroimage 2008; 40 : 1738-1747.

25 Kannurpatti SS, Biswal BB: Detection and scaling of task-induced fMRI-BOLD response using resting state fluctuations. Neuroimage 2008;40:1567-1574.

26 Zhao F, Zhao T, Zhou L, Wu Q, Hu X: BOLD study of stimulation-induced neural activity and resting-state connectivity in medetomidine-sedated rat. Neuroimage 2008;39:248260.

27 Hutchison RM, Mirsattari SM, Jones CK, Gati JS, Leung LS: Functional networks in the anesthetized rat brain revealed by independent component analysis of resting-state FMRI. J Neurophysiol 2010;103:3398-3406.

-28 Kalthoff D, Seehafer JU, Po C, Wiedermann D, Hoehn M: Functional connectivity in the rat at 11.7 T: Impact of physiological noise in resting state fMRI. Neuroimage 2011;54:2828-2839.

-29 Lu H, Zou Q, Gu H, Raichle ME, Stein EA, Yang Y: Rat brains also have a default mode network. Proc Natl Acad Sci USA 2012;109: 3979-3984.

30 Gass N, Schwarz AJ, Sartorius A, Cleppien D, Zheng L, Schenker E, Risterucci C, Meyer-Lindenberg A, Weber-Fahr W: Haloperidol modulates midbrain-prefrontal functional connectivity in the rat brain. Eur Neuropsychopharmacol 2013;23:1310-1319.

- 31 Becerra L, Pendse G, Chang PC, Bishop J, Borsook D: Robust reproducible resting state networks in the awake rodent brain. PLoS One 2011;6:e25701.

- 32 Liang Z, King J, Zhang N: Uncovering intrinsic connectional architecture of functional networks in awake rat brain. J Neurosci 2011;31: 3776-3783.

33 Liang Z, King J, Zhang N: Intrinsic organization of the anesthetized brain. J Neurosci 2012; 32:10183-10191.

- 34 Upadhyay J, Baker SJ, Chandran P, Miller L, Lee Y, Marek GJ, Sakoglu U, Chin CL, Luo F, Fox GB, Day M: Default-mode-like network activation in awake rodents. PLoS One 2011; 6:e27839.

-35 Zhang N, Rane P, Huang W, Liang Z, Kennedy D, Frazier JA, King J: Mapping resting-state brain networks in conscious animals. J Neurosci Methods 2010;189:186-196.

- 36 Monti MM, Lutkenhoff ES, Rubinov M, Boveroux $\mathrm{P}$, Vanhaudenhuyse A, Gosseries $\mathrm{O}$, Bruno MA, Noirhomme Q, Boly M, Laureys S: Dynamic change of global and local information processing in propofol-induced loss and recovery of consciousness. PLoS Comput Biol 2013; 9:e1003271.
37 Williams KA, Magnuson M, Majeed W, LaConte SM, Peltier SJ, Hu X, Keilholz SD: Comparison of $\alpha$-chloralose, medetomidine and isoflurane anesthesia for functional connectivity mapping in the rat. Magn Reson Imaging 2010; 28:995-1003.

38 Nasrallah FA, Tan J, Chuang KH: Pharmacological modulation of functional connectivity: $\alpha_{2}$-adrenergic receptor agonist alters synchrony but not neural activation. Neuroimage 2012; 60:436-446.

39 Jonckers E, van Audekerke J, de Visscher G, van der LA, Verhoye M: Functional connectivity fMRI of the rodent brain: comparison of functional connectivity networks in rat and mouse. PLoS One 2011;6:e18876.

40 Adamczak JM, Farr TD, Seehafer JU, Kalthoff D, Hoehn M: High field BOLD response to forepaw stimulation in the mouse. Neuroimage 2010;51:704-712.

41 Weber R, Ramos-Cabrer P, Wiedermann D, van Camp N, Hoehn M: A fully noninvasive and robust experimental protocol for longitudinal fMRI studies in the rat. Neuroimage 2006; 29:1303-1310.

42 van Buuren M, Gladwin TE, Zandbelt BB, van den Heuvel M, Ramsey NF, Kahn RS, Vink M: Cardiorespiratory effects on default-mode network activity as measured with fMRI. Hum Brain Mapp 2009;30:3031-3042.

-43 Cordes D, Haughton VM, Arfanakis K, Carew JD, Turski PA, Moritz CH, Quigley MA, Meyerand ME: Frequencies contributing to functional connectivity in the cerebral cortex in 'resting-state' data. AJNR Am J Neuroradiol 2001;22:1326-1333.

44 Cordes D, Haughton VM, Arfanakis K, Carew JD, Turski PA, Moritz CH, Quigley MA, Meyerand ME: Frequencies contributing to functional connectivity in the cerebral cortex in 'resting-state' data. AJNR Am J Neuroradiol 2001;22:1326-1333.

45 Boubela RN, Kalcher K, Huf W, Kronnerwetter C, Filzmoser P, Moser E: Beyond noise: using temporal ICA to extract meaningful information from high-frequency fMRI signal fluctuations during rest. Front Hum Neurosci 2013;7: 168.

-46 Lee HL, Zahneisen B, Hugger T, LeVan P, Hennig J: Tracking dynamic resting-state networks at higher frequencies using MR-encephalography. Neuroimage 2013;65:216-222.

-47 Margulies DS, Bottger J, Long X, Lv Y, Kelly C, Schafer A, Goldhahn D, Abbushi A, Milham MP, Lohmann G, Villringer A: Resting developments: a review of fMRI post-processing methodologies for spontaneous brain activity. MAGMA 2010;23:289-307.

48 Kanowski M, Kaufmann J, Braun J, Bernarding J, Tempelmann C: Quantitation of simulated short echo time ${ }^{1} \mathrm{H}$ human brain spectra by LCModel and AMARES. Magn Reson Med 2004; 51:904-912.

49 Provencher SW: Estimation of metabolite concentrations from localized in vivo proton NMR spectra. Magn Reson Med 1993;30:672-679.
50 Weber-Fahr W, Ende G, Braus DF, Bachert P, Soher BJ, Henn FA, Buchel C: A fully automated method for tissue segmentation and CSFcorrection of proton MRSI metabolites corroborates abnormal hippocampal NAA in schizophrenia. Neuroimage 2002;16:49-60.

51 Sartorius A, Henn FA, Ende G: Proton magnetic resonance spectroscopy as a monitoring tool for electroconvulsive therapy effects on the brain. Curr Psychiatry Rev 2006;2:39-49.

-52 Sartorius A, Lugenbiel P, Mahlstedt MM, Ende G, Schloss P, Vollmayr B: Proton magnetic resonance spectroscopic creatine correlates with creatine transporter protein density in rat brain. J Neurosci Methods 2008;172:215-219.

53 Moonen CT, van Zijl PC: Highly effective water suppression for in vivo proton NMR spectroscopy (DRYSTEAM). J Magn Reson 1990;88: 28-41.

- 54 Sartorius A, Mahlstedt MM, Vollmayr B, Henn FA, Ende G: Elevated spectroscopic glutamate/ $\gamma$-amino butyric acid in rats bred for learned helplessness. Neuroreport 2007;18:1469-1473.

- 55 Yang D, Xie Z, Stephenson D, Morton D, Hicks CD, Brown TM, Sriram R, O’Neill S, Raunig D, Bocan T: Volumetric MRI and MRS provide sensitive measures of Alzheimer's disease neuropathology in inducible Tau transgenic mice (rTg4510). Neuroimage 2011;54:2652-2658.

56 Goveas J, Xie C, Wu Z, Douglas WB, Li W, Franczak MB, Jones JL, Antuono PG, Yang Z, Li SJ: Neural correlates of the interactive relationship between memory deficits and depressive symptoms in nondemented elderly: resting fMRI study. Behav Brain Res 2011;219:205212.

57 Hasler G, Northoff G: Discovering imaging endophenotypes for major depression. Mol Psychiatry 2011;16:604-619.

58 Zhou Y, Shu N, Liu Y, Song M, Hao Y, Liu H, Yu C, Liu Z, Jiang T: Altered resting-state functional connectivity and anatomical connectivity of hippocampus in schizophrenia. Schizophr Res 2008;100:120-132.

59 Tye KM, Deisseroth K: Optogenetic investigation of neural circuits underlying brain disease in animal models. Nat Rev Neurosci 2012;13: 251-266.

60 Lee JH, Durand R, Gradinaru V, Zhang F, Goshen I, Kim DS, Fenno LE, Ramakrishnan C, Deisseroth K: Global and local fMRI signals driven by neurons defined optogenetically by type and wiring. Nature 2010;465:788-792.

61 Ashburner J: A fast diffeomorphic image registration algorithm. Neuroimage 2007;38:95113.

62 Biedermann S, Fuss J, Zheng L, Sartorius A, Falfán-Melgoza C, Demirakca T, Gass P, Ende $\mathrm{G}$, Weber-Fahr W: In vivo voxel based morphometry: detection of increased hippocampal volume and decreased glutamate levels in exercising mice. Neuroimage 2012;61:1206-1212.
Advantages and Challenges of Small Animal MRI
Neuropsychobiology 2014;69:187-201 DOI: $10.1159 / 000360859$ 